\title{
Appropriation différenciée de la démarche des cercles d'auteurs par quatre enseignantes du primaire
}

\author{
Auteur(s) \\ Ophélie Tremblay, Université du Québec à Montréal, Canada, \\ Tremblay.ophelie@uqam.qc.ca \\ Elaine Turgeon, Université du Québec à Montréal, Canada, \\ Turgeon.elaine@uqam.qc.ca \\ Brigitte Gagnon, Commission scolaire des Hautes-Rivières, Canada, \\ Brigitte.gagnon@csdhr.qc.ca
}




\section{REVUE HYBRIDE DE L'ÉDUCATION}

\section{Résumé}

Cet article présente les résultats d'une recherche-action visant à documenter la mise en œuvre des cercles d'auteurs par quatre enseignantes du primaire. En nous appuyant sur l'analyse de pratiques déclarées (entrevues, partages de pratiques, fiches de compte-rendu de pratiques), recueillies lors de la première année de mise à l'essai de la démarche, nous décrivons les pratiques différenciées de quatre enseignantes. Les résultats recueillis nous conduisent à montrer la pertinence des cercles d'auteurs comme approche d'enseignement et d'apprentissage de l'écriture, en illustrant comment celle-ci entraine des changements de pratiques importants en enseignement de l'écriture, au service du développement de la compétence à écrire des élèves.

Mots-clés: cercles d'auteurs; processus d'écriture; pratiques d'enseignement de l'écriture; enseignement primaire 


\section{$\&$}

\section{REVUE HYBRIDE DE L'ÉDUCATION}

\section{Les cercles d'auteurs : pratiquer et enseigner l'écriture autrement}

L'enseignement de l'écriture est marqué par la tradition scolaire et on rencontre encore dans les classes du primaire des pratiques qui mettent davantage l'accent sur la maitrise du code que sur l'écriture comme processus et pratique créative (Bucheton, 2014; Graham, McKeown, Kiuhara et Harris, 2012; Lafont-Terranova, 2009; Laroui, Morel et Leblanc, 2014). Privilégier le processus d'écriture dans son ensemble et valoriser la part créative de l'acte d'écrire permettrait pourtant aux élèves de mieux comprendre toute la complexité de l'écriture et la façon dont les auteurs de textes procèdent lorsqu'ils écrivent (Bucheton, 2014). C'est précisément le propos de démarches d'enseignement renouvelées de l'écriture comme les ateliers d'écriture (Calkins, 2017) et les cercles d'auteurs (Tremblay et Turgeon, 2019 ; Vopat, 2009), tel que nous l'avons fait valoir dans le texte de présentation de ce numéro. De telles démarches d'enseignement favorisent également une interaction forte entre les compétences langagières, notamment la lecture et l'écriture (Harste, Short et Burke, 1988; Le Goff et Larrivé, 2018; Tauveron, 2002), à travers des modalités interactives où la classe se transforme progressivement en véritable communauté d'auteurs et de lecteurs (Lebrun, 2007 ; Shea, 2015 ; Tauveron, 2007).

Dans une recherche-action menée conjointement avec des enseignantes du primaire entre 2016 et 2019, nous avons voulu expérimenter la démarche des cercles d'auteurs comme réponse à des besoins de développement professionnel en enseignement de l'écriture ${ }^{1}$. Plus précisément, nous avons fait l'hypothèse qu'en faisant vivre l'expérience des cercles d'auteurs aux enseignantes, elles seraient mieux à même de transposer la démarche en classe et de développer pour ellesmêmes des attitudes, des habiletés et des connaissances relatives à l'écriture et son enseignement. À cet effet, les travaux de recherche ayant porté sur la question des pratiques d'écriture des enseignants montrent que dans les classes des enseignants qui déclarent avoir une pratique d'écriture personnelle, les élèves réussissent mieux que ceux des classes dans lesquelles les enseignantes n'écrivent pas (Whyte et al., 2007). De plus, les enseignants qui entretiennent une perception d'eux-mêmes comme auteurs transmettent leur intérêt pour l'écriture à leurs élèves et cela teinte la façon dont ils l'enseignent (Gilbert et Graham, 2010). Or, une recherche québécoise récente (Lamb, Plante et Tremblay, 2017) montre que les enseignants écrivent peu, particulièrement dans une perspective créative, d'où la pertinence de notre projet de recherche-action, visant à conduire des enseignantes à faire l'expérience de l'écriture au fil de la démarche d'accompagnement et d'enseignement de l'écriture proposée dans le projet.

\footnotetext{
${ }^{1}$ Pour plus de détails sur l'évolution du développement professionnel des enseignantes participant au projet, voir l'article de Gagnon, Tremblay et Turgeon, dans ce numéro.
} 


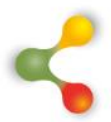

\section{REVUE HYBRIDE DE L'ÉDUCATION}

Dans cet article, nous souhaitons montrer comment quatre des enseignantes ayant participé au projet de recherche-action sur les cercles d'auteurs ont transposé en classe l'ensemble de la démarche lors de la première année de mise à l'essai en classe. Afin de mieux situer la description de la mise en œuvre différenciée de la démarche des cercles d'auteurs, nous commencerons par décrire les fondements théoriques et pratiques qui soutiennent la démarche, telle que présentée dans le texte d'introduction de ce numéro. Nous décrirons ensuite la méthodologie mise en œuvre afin de recueillir les données relatives à la mise à l'essai de la démarche par quatre des enseignantes participantes (deux au $2^{\mathrm{e}}$ cycle du primaire et deux au $3^{\mathrm{e}}$ cycle; élèves de 8 à 12 ans). La section consacrée aux résultats décrit les pratiques de chacune des enseignantes (lors de la première année du projet) au regard de différents éléments d'analyse (fréquence des cercles, modes de regroupement d'élèves, choix du sujet d'écriture, etc.), qui sont synthétisées et discutées dans la dernière partie de l'article. En conclusion, nous revenons sur les retombées de cette recherche-action quant au développement professionnel des enseignants en écriture et au renouvellement de leurs pratiques d'enseignement.

\section{Les fondements théoriques des cercles d'auteurs}

Les deux sections qui suivent présentent les éléments théoriques clés sur lesquels s'appuient notre dispositif de développement professionnel et nos résultats de recherche, soit: 1) la démarche des cercles d'auteurs telle que formalisée par Vopat (2009), ainsi que ses liens avec les concepts de posture d'auteur et de posture de lecteur et 2) les principes qui sous-tiennent la démarche - chacun étant appuyé sur le plan théorique par un certain nombre d'écrits scientifiques.

Les cercles d'auteurs : quand écrire devient un acte solidaire où posture d'auteur, posture de lecteur et posture d'écoute interagissent

Les cercles d'auteurs sont de petits groupes de participants (trois à cinq) qui écrivent fréquemment, sans contrainte de genre ou de thème, et qui partagent leurs productions, se donnent de la rétroaction sur cellesci, puis les révisent ensuite pour les publier (Vopat, 2009). La démarche se divise en plusieurs phases qui correspondent aux différentes étapes du processus d'écriture modélisé par Hayes et Flower en 1980 et mis à jour par Hayes, en 1995. Dans le cadre de notre projet, nous avons distingué quatre types de cercles (cercle de planification, de partage, de révision et de correction) $)^{2}$, étroitement liés aux phases du processus d'écriture.

La collaboration qui structure toutes les étapes de la démarche montre que la pratique des cercles d'auteurs s'appuie sur une vision socioconstructiviste de l'apprentissage de l'écriture (Bronckart, 2006) : les

\footnotetext{
${ }^{2}$ Voir le texte de présentation du numéro pour une présentation détaillée de chacun des cercles.
} 


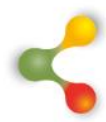

\section{REVUE HYBRIDE DE L'ÉDUCATION}

élèves travaillent systématiquement en petits groupes, du choix du thème au partage des textes, ce qui affirme l'aspect social des apprentissages réalisés dans ce type de démarche. En ce sens, les cercles d'auteurs correspondent à ce que Cairney et Langbien (1989) et Schultz (1997) appellent Writing Community. De telles communautés de scripteurs permettent aux élèves de faire l'expérience d'une posture d'auteur tout en contribuant à la création de classes où les élèves sont perçus et agissent comme des auteurs (Short, Harste et Burke,1996). Selon Bautier et Bucheton (1995) et Rebière (2001), dans un contexte didactique, la posture d'auteur correspond à une position énonciative qu'adopte un élève et à partir de laquelle il donnera un sens à une activité d'écriture donnée. La posture d'auteur se développerait à travers à une appropriation du processus d'écriture, notamment dans des situations d'écriture sortant du cadre traditionnel d'apprentissage de l'écriture (Docquet-Lacoste, Lumbroso et Tauveron, 2009).

L'appropriation d'une posture d'auteur conduit les élèves à une meilleure prise en compte des attentes du lecteur (Harwayne, 1992 ; May, 2004). Au sein des cercles d'auteurs, les élèves prennent systématiquement une posture de lecteur, puisqu'ils écoutent le texte d'un pair lors du cercle de partage, et sont amenés à relire un autre texte en vue de soutenir le processus de révision de son auteur, lors du cercle de révision. Cette posture de lecteur est susceptible de soutenir en retour le processus d'élaboration de textes, tout en contribuant à en améliorer la qualité (Blain, 2001 ; Blain et Painchaud, 1999). La mise en œuvre réussie de la démarche repose par conséquent sur la création d'une communauté d'auteurs-lecteurs (Lebrun, 2007; Tauveron et Sève, 2005) et sur la collaboration entre élèves pour soutenir les différentes étapes du processus d'écriture. L'oral intervient également tout au long de la démarche (Tremblay et Turgeon, 2019), notamment dans le développement d'une posture d'écoute (Dumais, 2012), tant au sein du cercle de planification (accueillir les idées des autres) que des cercles de partage (écouter le texte partagé avec attention et bienveillance) et de révision (l'auteur accueille les commentaires de ses pairs à propos de son texte).

\section{Les principes soutenant la démarche des cercles d'auteurs}

Les principes soutenant la démarche des cercles d'auteurs ont été dégagés, d'une part, à partir des écrits de référence sur les cercles d'auteurs (Gunnery, 2007; Vopat, 2009), et, d'autre part, à partir de l'expérience vécue en cercles d'auteurs par les enseignantes de notre projet lors des premières journées de formation en communauté d'apprentissage (un type de démarche de développement professionnel dont l'article de Gagnon, Tremblay et Turgeon, dans ce numéro, présente une caractérisation). Une série de huit principes a été établie conjointement avec les enseignantes. Chacun d'entre eux fait écho à des principes 


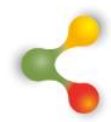

\section{REVUE HYBRIDE DE L'ÉDUCATION}

didactiques, des résultats de recherche ou des recommandations mises en évidence par les écrits en didactique de l'écriture.

\section{L'enseignant doit être un auteur}

Ce principe traverse tout notre projet. À l'instar d'E. Bing dans le cadre des ateliers d'écriture qu'elle a animés auprès des enfants, nous avons vécu les cercles d'auteurs avec les enseignantes, portées "par le désir de les faire écrire comme si [elles] devaient un jour devenir écrivains " (Bing, 1976, p. 4). Cette prise de position s'inscrit dans la foulée des recommandations formulées par plusieurs chercheurs concernant l'importance, pour l'enseignant, d'avoir des pratiques d'écriture personnelles (Graves, 2004 ; Grossman et al., 2000 ; McDonald, Buchanan et Sterling, 2004 :; Nadon, 2011 ; Oriol-Boyer, 1980 et Routman, 2010). Faire l'expérience des batailles et des satisfactions du travail de l'écrivain outillerait efficacement les enseignants pour accompagner leurs élèves dans l'apprentissage de l'écriture (Blau, 1988; Brooks, 2007; Cremin, 2006). De plus, lorsque les enseignants ont une perception d'eux-mêmes comme auteurs, cela influence positivement leur façon d'enseigner l'écriture et les expériences qu'ils font vivre aux élèves (Gilbert et Graham, 2010 ; Harward et al., 2014). Le fait pour les enseignants d'adopter une posture d'auteur favorise enfin l'adoption d'une posture de lecteur (plutôt que d'évaluateur) lorsqu'ils ont ensuite à lire les productions de leurs élèves (Sorin, 2005).

\section{L'écriture doit faire partie de la routine de classe}

Afin de développer leur confiance en eux, leur motivation et une certaine souplesse dans l'acte d'écrire, les élèves ont besoin de prendre la plume le plus souvent possible, idéalement tous les jours. En écrivant quotidiennement, animés de diverses intentions et en ayant en tête de vrais destinataires, ils peuvent construire leur compétence à écrire (Fletcher, 1993; Graves, 2004; Murray, 1989). La pratique de l'écriture libre (Clanché, 1988) s'inscrit aussi dans la perspective d'une routine de classe et plusieurs chercheurs ont mis en évidence son rôle dans l'amélioration de diverses facettes de la compétence à écrire (Murray, 1989 ; Ponsot et Deen, 1982 ; Rief, 2003, cités dans Routman, 2010).

\section{La démarche doit être enseignée}

Plusieurs travaux ont démontré que les interactions sociales facilitent la progression des apprentissages (Buchs, Darnon, Quiamzade, Mugny et Butera, 2008 ; Cohen et al., 2002; Slavin, 1980). Les cercles d'auteurs s'inscrivent dans ce courant et misent sur la collaboration entre élèves pour soutenir le processus d'écriture. Toutefois, pour que les élèves collaborent efficacement et progressent en écriture, il est indispensable que des savoirs de références aient été préalablement construits (Colognesi et Lucchini, 2018). Ainsi, bien que les cercles d'auteurs favorisent l'autonomie 


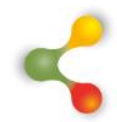

\section{REVUE HYBRIDE DE L'ÉDUCATION}

chez les élèves, l'enseignant a plusieurs rôles importants à jouer afin d'assurer le bon déroulement de la démarche. Un enseignement explicite de certains savoirs, savoir-faire ou savoir-être sera ainsi réalisé à travers les mini-leçons qui structurent la démarche. Les mini-leçons (Calkins, 2017 ; Rog, 2011) sont de courtes séances d'enseignement qui visent un objet bien précis, ciblé en réponse aux besoins ou défis rencontrés par les élèves lors des cercles. II peut s'agir, par exemple, d'enseigner, en lien avec le cercle de planification, comment trouver des idées et les organiser. Pour le cercle de partage, il pourrait s'agir d'enseigner à formuler un commentaire positif alors que pour le cercle de révision, les mini-leçons pourraient viser les habiletés suivantes : écouter le texte d'un pair, formuler un commentaire ou y réagir, aider un pair à mieux ponctuer son texte, etc.). En somme, plusieurs habiletés sociales et compétences à l'oral sont nécessaires au bon déroulement des cercles d'auteurs, comme le rappellent Daniels (2006) et Steineke (2002) pour les cercles de lecture.

\section{La créativité doit être au centre du travail d'écriture}

Reuter (1996) voit la créativité comme «[...] des mécanismes spécifiques de production (génération/transformation) de contenus. " (p.29) La créativité fait appel à la fabrication et à l'intentionnalité (Lekeuche, 2011), dans le but de trouver une solution inédite face à un problème en utilisant notre propre expression (Lamblin, 2012). Elle est également une façon d'expérimenter tout en se donnant le droit à l'erreur (Lamblin, 2012). L'écriture constitue avant tout un mode d'expression et non un travail mécanique effectué pour répondre à une consigne et obtenir une note. La liberté de choisir son sujet et son destinataire soutient en ce sens la part créative de chaque élève et contribue à la construction d'un scripteur qui aime écrire (Graves, 1994 ; Routman, 2010).

5. L'évaluation doit porter aussi bien sur le processus que sur le produit final

Quand l'évaluation se fait en cours d'apprentissage et porte sur les différentes étapes du processus plutôt que sur la seule production finale, l'élève est plus susceptible de s'améliorer et de concevoir l'écriture comme un processus qui se déroule dans le temps et non dans la durée restreinte d'une production écrite sur commande (Cotton, 1988 ; Graves, 1983 ; Sorin, 2005). À cet égard, le fait de conserver les copies des élèves (avant et après la rencontre en cercle d'auteurs) permet à l'enseignant de voir les idées notées en cercle de planification, d'observer les annotations faites sur son premier jet, lors du cercle de révision ${ }^{3}$, et de constater les améliorations apportées au produit final. Cela permet enfin à l'enseignante de juger de l'évolution de la production écrite et de la compétence à écrire dans son ensemble (Le Goff, 2018).

\footnotetext{
${ }^{3}$ Pour en savoir davantage sur le travail de révision des élèves en cercle d'auteurs, consulter l'article de Turgeon, Tremblay et Gagnon, dans le présent numéro.
} 


\section{6}

\section{REVUE HYBRIDE DE L'ÉDUCATION}

\section{La littérature jeunesse doit nourrir le processus d'écriture}

Nourrir et enrichir le capital culturel et littéraire des élèves est une des valeurs clés qui soutient la démarche des cercles d'auteurs. Les livres représentent également une source d'inspiration et peuvent être utilisés comme déclencheurs pour l'écriture : écrire à partir de la page couverture d'un album, écrire pour imaginer la suite du récit, écrire une nouvelle fin à une histoire, réécrire l'histoire en adoptant un point de vue de narration différent, etc. L'observation des procédés littéraires employés par les auteurs vient également soutenir la démarche et contribue à construire la posture d'auteur et de lecteur de l'élève. Le regard sur la lecture est modifié : l'élève apprend à lire comme un écrivain (Griffith, 2010 ; OriolBoyer, 1980 ; Ray, 2000). La littérature de jeunesse joue ainsi un rôle crucial dans l'apprentissage de «l'art de l'écriture »(Olness, 2005).

\section{Le respect est primordial}

Un des ingrédients essentiels de la démarche des cercles d'auteurs est le respect. Écrire est un acte personnel qui engage l'affectif autant que le cognitif. Instaurer un climat de confiance qui encourage les élèves à prendre des risques sans craindre l'échec est donc un gage de succès (Cohen et al., 2002 ; Routman, 2010). Cette valeur incontournable s'inscrit également dans la philosophie des communautés de lecteursauteurs (Lebrun, 2007 ; Tauveron et Sève, 2005). Chacune des étapes, de la planification à la publication, doit être vécue dans ce climat de respect et de bienveillance. C'est ce que met en évidence l'article de Rainville, dans ce numéro, qui illustre comment construire un climat propice à la coopération.

\section{La rétroaction doit être constructive}

Dans la même veine, les élèves doivent apprendre à formuler des remarques constructives qui aideront l'auteur à améliorer son texte (OriolBoyer, 1986). Les mini-leçons sont ici indispensables pour apprendre aux élèves à formuler de telles remarques, qui contribueront d'ailleurs au maintien d'un fonctionnement harmonieux au sein des cercles (Cohen et al., 2002). Par exemple, le cercle de partage permet aux élèves de recevoir des commentaires concernant le message livré par leur texte. II s'agit donc pour les pairs d'exprimer ce que suscite le texte en eux, par exemple : « J'ai adoré la fin de ton texte. Elle m'a surpris! » Le cercle de révision permet ensuite de formuler des commentaires qui visent à améliorer le texte : «Je ne suis pas certaine de comprendre ce passage. Que veux-tu dire quand tu dis...? » Dans tous les cas, les commentaires doivent être précis et permettre à l'auteur d'en tirer profit, au service de l'amélioration du texte (Simard, 1999). 


\section{$\&$}

\section{REVUE HYBRIDE DE L'ÉDUCATION}

L'objet de cet article est de montrer comment, lors de la première année de mise à l'essai, les enseignantes de notre projet se sont approprié à la fois la démarche des cercles d'auteurs et les principes qui la soustendent.

\section{Méthodologie}

\section{Participants}

Comme nous l'avons expliqué dans le texte d'introduction de ce numéro, nous avons misé sur la création d'une communauté d'apprentissage (Dionne, Lemyre et Savoie-Zajc, 2010) pour mener notre recherche. Cette communauté est formée d'enseignantes ${ }^{4}$ de deux écoles de la Commission scolaire des Hautes-Rivières, au Québec, ainsi que de deux orthopédagogues. Une équipe d'encadrement et d'accompagnement du projet fait également partie de cette communauté d'apprentissage. Elle est composée de deux chercheuses, d'une cochercheuse du milieu de pratique qui œuvre comme conseillère pédagogique, d'une directrice d'école, d'une orthopédagogue et d'un conseiller pédagogique en français.

\section{Déroulement}

La recherche-action s'est déroulée entre avril 2016 et juin 2018. Au fil de ces deux années, les activités de formation ont été articulées autour d'objectifs de développement professionnel correspondant aux besoins des enseignants, d'une part, et aux objectifs du projet, d'autre part. Le développement d'une posture d'auteure chez les enseignantes a constitué une cible de formation tout au long du projet. Le développement de connaissances en écriture (planification, révision) a par la suite été travaillé durant les journées de suivi. Pour atteindre ces cibles de formation, deux camps littéraires ont été organisés (août 2016, août 2017) et les enseignantes ont participé à une dizaine de rencontres de formation et de suivi lors desquelles elles ont systématiquement vécu des cercles d'auteurs entre elles.

\section{Outils de collecte de données et procédure d'analyse}

Des instruments variés ont été utilisés au fil du projet afin de recueillir les données concernant la mise en œuvre des cercles d'auteurs : entrevues semi-dirigées, journal de bord, bilan réflexif, transcriptions de partages lors des journées de formation et de suivi, documents produits par les enseignantes. Pour les fins de cet article, nous avons fait l'analyse du contenu de trois de ces instruments de collecte.

\footnotetext{
${ }^{4}$ Au départ de la recherche-action, sept enseignantes participaient à la communauté d'apprentissage. Trois d'entre elles ont quitté le projet pour des raisons professionnelles ou familiales.
} 


\section{$\&$}

\section{REVUE HYBRIDE DE L'ÉDUCATION}

D'abord, les entrevues semi-dirigées, menées à deux reprises (mai 2016, mai 2017) durant la première année du projet et abordant les thèmes suivants: le rapport à l'écriture des enseignantes, l'évolution de leur posture d'auteur à travers les cercles d'auteurs vécus dans la communauté d'apprentissage, leurs pratiques personnelles d'écriture et leurs pratiques d'enseignement de l'écriture, incluant l'expérimentation des cercles d'auteurs en classe.

Ensuite, les journaux de bord dans lesquels la chercheuse principale et son assistante avaient consigné les observations relatives è la mise en œuvre de la démarche, aux bons coups et difficultés partagés par les enseignantes, en plus de questionnements et de contenu à planifier en vue des rencontres suivantes.

Enfin, les bilans réalisés par les enseignantes en mai 2017 après la première année de mise à l'essai des cercles d'auteurs. Ces bilans comportent des lignes du temps de la mise en œuvre des cercles par chacune des enseignantes: activités d'écriture réalisées chaque mois, type de cercle mis à l'essai, synthèse des bons coups et des défis rencontrés par elles-mêmes et par leurs élèves.

L'analyse de ces données a été menée à travers une approche mixte. Une analyse en mode écriture (Paillé et Mucchielli, 2016) a d'abord été réalisée. Nous avons ainsi procédé à des lectures successives des entrevues des enseignants, de nos journaux de bord de recherche et des bilans produits par les enseignantes. Cet exercice nous a permis de dégager différents éléments récurrents parmi les données. Des aspects constitutifs de la démarche des cercles d'auteurs telle que mise en œuvre par les enseignantes ont émergé de ce travail d'analyse: mode de formation des équipes de cercles d'auteurs, question du choix du sujet d'écriture, modalités d'enseignement de la démarche, fréquence des cercles. De façon plus cadrée, nous avons eu recours à notre liste de principes phares de la démarche afin de voir comment ceux-ci se manifestaient dans les pratiques déclarées des enseignantes, en fonction, notamment du contenu d'enseignement, du processus d'apprentissage des élèves et de l'environnement de la classe (Shea, 2015). À partir des éléments issus de ces différents angles d'analyse, nous avons pu établir des portraits de chacune des enseignantes quant à leur mise en œuvre respective des cercles d'auteurs en classe.

\section{Résultats}

Nous présentons d'abord un tableau synthèse qui permet de faire ressortir, de façon graphique, les particularités de la mise en œuvre de la démarche (lors de la première année du projet) chez chacune des enseignantes en ce qui concerne : 


\section{8}

\section{REVUE HYBRIDE DE L'ÉDUCATION}

a) le nombre de cercles d'auteurs faits en classe au fil des mois de l'année scolaire, incluant les cercles faits sous la formule "aquarium ${ }^{5}$ (indiqués par un cercle en pointillé) ;

b) le nombre d'élèves dans les cercles $(2,4$, ou non spécifié - un cercle en aquarium se réalisant habituellement à 4 ou 5 élèves) ;

c) le choix libre ou imposé du sujet (indiqué par un triangle - plein pour le choix libre, vide pour le choix imposé, et en pointillé pour un sujet imposé par l'enseignante, mais comportant des choix).

Tableau 1 : Modalités de mise en œuvre des cercles d'auteurs (20162017)

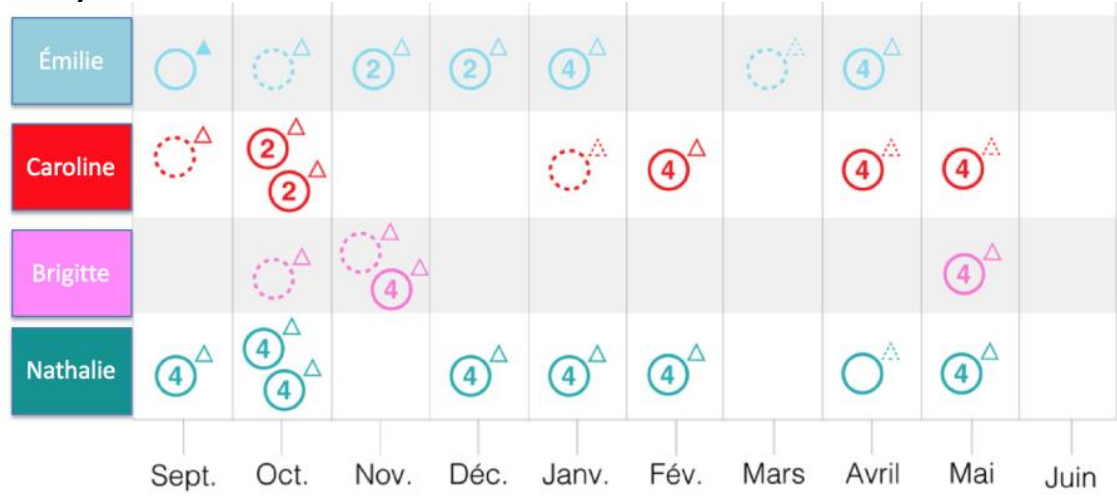

Au premier coup d'œil, on constate que les quatre enseignantes ont réalisé un minimum de quatre cercles d'auteurs durant l'année et un maximum de huit. La modalité la plus fréquente est le cercle à quatre élèves, avec un choix de sujet imposé. La formule aquarium a été utilisée par trois des enseignantes, au début de l'année en particulier, mais à au moins une autre reprise durant l'année, probablement pour introduire le fonctionnement du cercle de révision, qui demande une modélisation pour mieux maitriser la nature et la forme des commentaires à formuler afin de soutenir le processus de révision des pairs.

Voyons maintenant ce qui caractérise la mise en œuvre de chacune des enseignantes, telle que nous avons pu la dégager à partir de l'analyse des entrevues et des bilans de la première année du projet, autour des catégories suivantes : thèmes abordés lors des mini-leçons, fréquence

\footnotetext{
5 |l s'agit d'une modalité coopérative qui consiste à demander à un petit groupe d'élèves de venir au centre de la classe pour vivre une des étapes de la démarche (un cercle de planification, par exemple). Les autres élèves observent alors le déroulement de l'activité ou de la tâche afin de mieux en comprendre les caractéristiques. Voir l'article de Brigitte Rainville (ce numéro) pour plus de détails à propos de la formule de l'aquarium.
} 


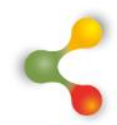

\section{REVUE HYBRIDE DE L'ÉDUCATION}

des activités d'écriture, apport de la littérature jeunesse, liens avec les principes phares de la démarche.

Caroline: Des pratiques d'écriture fréquentes et un enseignement explicite de la démarche pour susciter le plaisir d'écrire et développer la compétence scripturale.

Dès ses premières mises à l'essai des cercles d'auteurs, au début de l'année scolaire 2016-2017, Caroline, enseignante en $4^{\circledR}$ année (élèves de 9-10 ans), fonctionne avec des équipes de quatre ou cinq élèves, en fonction des forces et faiblesses de chacun. Elle bénéficie de l'aide de l'orthopédagogue en classe, lorsqu'elle organise des cercles d'auteurs, ce qui facilite sa mise à l'essai.

Son travail d'observation, lors de la tenue des cercles, lui permet d'évaluer les connaissances et savoir-faire des élèves en écriture. Elle s'en sert ensuite pour planifier ses contenus d'enseignement à travers des minileçons :

Par exemple, dans une équipe, ils avaient retravaillé une phrase complètement parce qu'ils n'avaient pas compris le sens, et il y avait des mots qui étaient un petit peu ternes. Je m'étais assise avec eux et j'avais pris en note ce qu'ils avaient noté dans le texte et comment ils avaient fait pour retravailler ça. Donc j'ai pris la version « avant/après » et j'ai intégré ça en mini-leçon en grand groupe. Ça a porté fruit parce que, par la suite, je l'ai remarqué plusieurs fois dans les autres cercles.

En réponse à ce qu'elle observe dans les cercles, elle travaille en profondeur la question de la formulation des commentaires sur les textes, que ce soit pour les cercles de partage ou pour les cercles de révision. Pour ces derniers, par exemple, elle utilise le tableau numérique interactif, sur lequel elle projette le texte de l'élève et illustre comment en réviser certains aspects, sous la forme d'une mini-leçon. Ces moments contribuent à une meilleure compréhension des étapes du processus d'écriture chez les élèves, qui réalisent qu'il existe une différence entre l'écriture d'un texte, la révision et la correction de celui-ci. Suivant le principe selon lequel la démarche doit être enseignée, Caroline se pose également en modèle pour aborder certains procédés d'écriture : “ Je m'étais lancée, moi-même... Et ça a donné quelque chose d'intéressant avec les élèves parce qu'ils ont repris certains éléments qu'on avait travaillés ensemble. »En entrevue, interrogée à propos des changements de pratiques introduits à travers la démarche des cercles d'auteurs, elle nous dit : «C'est plutôt au niveau du contenu plutôt que du contenant ce que j'enseigne maintenant. Voici comment on peut arriver à décrire, comment on peut améliorer certains passages. 》 


\section{$\&$}

\section{REVUE HYBRIDE DE L'ÉDUCATION}

Ce qui se dégage de son bilan, en plus d'un enseignement plus explicite, c'est la fréquence des activités d'écriture mises en place tout au long de l'année scolaire (principe 2 de la démarche), une pratique qui contraste avec ce qu'elle avait l'habitude de faire auparavant: "Je demandais environ deux fois par année aux élèves d'écrire des textes. » Par les nombreux jeux d'écriture qu'elle propose à ses élèves, elle souhaite leur transmettre le plaisir qu'elle a éprouvé elle-même en vivant les cercles d'auteurs dans le dispositif d'accompagnement professionnel. C'est d'ailleurs le souhait qu'elle avait formulé au terme des quatre premières journées de formation, au printemps 2016 :

Le fait d'avoir du plaisir à écrire, j'espère que je vais être capable de le transmettre à mes élèves, pour qu'eux aussi aient du plaisir à écrire. Je comprends mieux mes élèves maintenant, je suis plus capable de me mettre dans leur peau, comprendre leurs craintes, leur déplaisir.

On constate ici une adhésion forte au principe selon lequel l'enseignant doit être un auteur et se poser en modèle.

Émilie : Approfondir l'enseignement de l'écriture et du processus de révision tout en questionnant les pratiques d'évaluation et en les renouvelant

La mise en place des cercles d'auteurs s'est faite de façon progressive dans la classe d'Émilie, enseignante en $6^{e}$ année (élèves de 11-12 ans). Son expérimentation spontanée d'un cercle d'auteur avec sujet libre, en début d'année scolaire, ne donne pas les résultats escomptés et elle reviendra à un enseignement en grand groupe et à de nombreuses modélisations, à travers des mini-leçons, avant de faire revivre un cercle d'auteurs à ses élèves.

Moi, le choix du thème, je l'ai expérimenté une fois, puis ça complètement dérapé. Tout ce qui était nommé là, c'était, surtout par les garçons, ce n'était que pour faire rire. Donc ç'a donné des textes qui... "Pfff». Est-ce qu'il faudrait modeler ou parler un peu de ce qu'est un bon sujet, de ce qui peut faire l'objet d'un bon texte, pour justement encadrer un peu le choix du thème?

Cette expérience illustre le besoin de modéliser devant les élèves ce qu'est un bon sujet, et comment faire émerger des idées. La planification, comme tout objet de savoir en écriture, doit être enseignée pour que les élèves s'approprient pleinement cette étape du processus d'écriture. En ce sens, la pratique d'Émilie, tout au long de l'année, s'inscrit de plain-pied dans le principe 3 . Les situations d'écriture fréquentes et variées qu'elle a fait vivre à ses élèves tout au long de l'année lui permettent en effet d'aborder différentes connaissances et savoir-faire en écriture : 


\section{$\&$}

\section{REVUE HYBRIDE DE L'ÉDUCATION}

comment trouver des idées, s'inspirer d'images pour écrire, raconter une anecdote, utiliser la comparaison, faire appel aux cinq sens pour rendre un texte plus vivant, etc. Elle travaille également certains genres textuels en profondeur (la lettre, le récit, l'affiche publicitaire). La fréquentation d'œuvres de littérature de jeunesse soutient quant à elle le processus d'écriture des élèves, les œuvres servant de déclencheurs d'écriture ou de lieu d'observation de l'art d'écrire, tel que le stipule le principe 6.

La lecture des textes produits par les élèves, quant à elle, ne se fait pas dès le départ en cercles de partage, mais en grand groupe. Émilie mise sur cette façon de faire pour enseigner explicitement les attitudes à privilégier lors du partage d'un texte, tant du point de vue de l'auditeur (posture d'écoute, respect, formulation de commentaires positifs, etc.) que du lecteur (ne pas commenter son texte avant d'en faire la lecture, lire suffisamment fort, bien prononcer les mots, rendre sa lecture expressive, etc.).

En somme, cette première année d'expérimentation, pour elle, se caractérise par la présence de très nombreuses activités d'écriture, exploitant des genres variés, par un enseignement explicite des composantes du processus d'écriture et de la démarche elle-même.

Si Émilie a vécu de nombreuses expériences positives en enseignement de l'écriture au fil de cette année d'expérimentation, la question de la révision apparait néanmoins comme un enjeu central au fil de l'expérience des cercles d'auteurs dans sa classe. Ce questionnement à propos de l'étape de la révision et les interrogations à propos des manières renouvelées d'évaluer les productions des élèves à travers la démarche joueront le rôle de leviers pour la deuxième année d'expérimentation. Durant celle-ci, Émilie affinera son enseignement de la révision et adaptera ses pratiques d'évaluation en fonction d'une démarche plus souple de la production d'écrits chez ses élèves (voir article de Bhérer dans ce numéro).

Brigitte : De l'importance d'un climat de classe positif pour une mise en œuvre réussie des cercles d'auteurs

Brigitte enseigne en $5^{e}$ année (élèves de 10-11 ans). L'écriture prend déjà une grande place dans la vie de sa classe et sa première année d'expérimentation s'inscrit donc sous le signe de la continuité, mais aussi d'une plus grande fréquence et variété de jeux d'écriture. Dès le début de l'année, elle anime des activités qui vont nourrir les valeurs coopératives qui soutiennent la vie de la classe et qu'elle considère comme des éléments clés de la démarche même des cercles d'auteurs (principe 7). Comme elle l'explique dans l'article qu'elle signe dans ce numéro, Brigitte fait appel à la formule de l'aquarium pour enseigner la démarche. Une fois la modélisation en aquarium effectuée, les élèves sont placés en équipe de deux pour vivre 


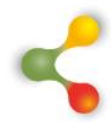

\section{REVUE HYBRIDE DE L'ÉDUCATION}

les activités d'écriture en classe. Elle choisit cette adaptation pour maximiser le déroulement du processus d'écriture.

En aquarium, ils sont quatre, parce que je modélise. Après ça là, dans la réalité, c'est deux. C'est ce qui va le mieux jusqu'à présent, parce qu'on l'a fait déjà là, mais je fais plus de gestion de comportements que d'autre chose...

Un peu plus tard dans l'année elle remarquera tout de même que les équipes peuvent être composées d'élèves de forces complémentaires : «les équipes hétérogènes, je trouve que c'est plus riche... »

Les cercles de partage se font quant à eux à travers la formule de l'aquarium. Progressivement, Brigitte installe la "minute de l'auteur », un moment où un élève partage un texte devant toute la classe. En plus de contribuer à affirmer la posture d'auteur des élèves, cette façon de faire est plus économique, en termes de temps et de gestion de classe, que les cercles de partage. Elle permet également à Brigitte d'enseigner les comportements attendus de la part des auditeurs et de l'auteur qui lit son texte devant ses camarades et de construire un climat de confiance entre les élèves-auteurs.

Brigitte recourt systématiquement à la littérature jeunesse dans son enseignement (principe 6) : “On regarde les procédés d'auteurs qu'il y a dans les albums, après ça les élèves essaient de les appliquer, en les utilisant dans leurs propres textes. »Elle fera également venir en classe un auteur pour la jeunesse et une poète, visites qui stimuleront son enseignement de l'écriture et la créativité de ses élèves. Son évaluation de la compétence à écrire des élèves porte par conséquent, au fil de l'année, sur les procédés observés dans les œuvres: "Quand j'arrive à l'évaluation, je mets des étoiles à côté des procédés qu'ils ont utilisés. Je me suis rendu compte qu'ils en avaient tous utilisés! » Elle s'adapte également, dans son processus d'évaluation, à l'évolution de chaque élève scripteur (principe 5) : «II faut de l'adaptation, à partir de ce qu'on lit des élèves. Faut être prêt aussi à ne pas évaluer tout de suite. Accepter que ça prenne du temps, accepter qu'ils ont besoin d'intégrer... »

En somme, les résultats observés chez les élèves contribuent à nourrir l'enthousiasme de Brigitte et son engagement envers l'écriture et la démarche des cercles d'auteurs, même si elle n'en fait pas un usage systématique dans la forme initiale durant cette première année de mise en œuvre.

Nathalie: Les cercles d'auteurs pour passer de l'attention sur le code à une valorisation du processus d'écriture dans son ensemble

Enseignante en $6^{\mathrm{e}}$ année, Nathalie fait déjà beaucoup de place à l'enseignement de l'écriture dans sa classe, en se consacrant cependant 


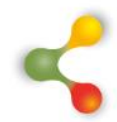

\section{REVUE HYBRIDE DE L'ÉDUCATION}

davantage à la question de la maitrise du code, du moins au départ du projet. Progressivement, elle intègre des aspects liés au processus dans son ensemble (principe 5) et elle plonge avec beaucoup d'enthousiasme dans la mise à l'essai de la démarche. Dès le début de l'année scolaire 2016-2017, elle met en place les cercles d'auteurs, avec la formule de l'aquarium. Lorsque les cercles d'auteurs ont lieu, elle rapporte observer chez les élèves des comportements qui ont fait l'objet d'un enseignement préalable: respect, pistes pour la recherche d'idées, propositions créatives, procédés d'auteurs variés. Quant au choix du sujet, celui-ci est imposé (même si elle offre des choix, parmi les sujets soumis) lors de chacun des cercles d'auteurs vécus en classe.

Nathalie modélise beaucoup son propre processus d'écriture et fait appel, elle aussi, à la littérature jeunesse comme moteur pour l'écriture (principe 6). Son coup de cœur pour la liberté créative que lui ont apporté les cercles d'auteurs vécus en communauté d'apprentissage se propage dans sa classe et elle découvre avec bonheur la fibre créative de ses propres élèves à travers les textes produits au fil de l'année :

On s'attend à ce que les plus forts continuent à être les plus forts, bien sûr. Mais ceux qui avaient des petits textes, des courts textes et qui n'avaient plus d'idées après quatre lignes, là, et qui ont eu de l'aide avec d'autres élèves, puis qui ont dû, deux fois, trois fois, lire leurs textes parce que y'avait le partage, la révision... ils se sont rendu compte qu'ils avaient des idées en dedans d'eux et qu'il fallait les placer sur la feuille! Ça fait qu'ils ont réussi à améliorer leurs textes:

Le sentiment de fierté éprouvé par Nathalie devant l'évolution de la compétence scripturale des élèves est aussi partagé par les autres enseignantes du projet, qui rapportent constater de grands progrès en écriture chez tous leurs élèves. L'adhésion forte des enseignantes au principe selon lequel il est important de valoriser l'écriture comme processus représente selon nous un des facteurs expliquant les progrès constatés par les enseignantes chez leurs élèves.

\section{Discussion}

Les portraits présentés illustrent les retombées d'une année d'expérimentation des cercles d'auteurs en classe (2016-2017) sur le développement professionnel des enseignantes en écriture et sur le renouvellement des pratiques de classe. Certains points de convergence émergent de notre analyse, concernant l'appropriation de la démarche par les enseignantes, le type de difficultés rencontrées et l'adhésion aux grands principes de la démarche. 


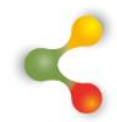

\section{REVUE HYBRIDE DE L'ÉDUCATION}

Une première année d'expérimentation entre réussites, tensions et réflexions

Rappelons qu'au départ de la mise à l'essai en classe, les enseignantes ont vécu elles-mêmes des cercles d'auteures, suivant le principe de l'approche expérientielle (Kolb, 1984). Elles connaissent ainsi la démarche «de l'intérieur» et en maitrisent les étapes (cercle de planification, cercle de partage, cercle de révision, cercle de publication). Comme chercheuses et accompagnatrices, nous faisions l'hypothèse que ces connaissances et savoir-faire seraient suffisants pour mettre en œuvre la démarche dans leur classe, dès le début de l'année scolaire 2016-2017. Les échanges vécus lors des journées de suivi pendant cette première année d'expérimentation ont cependant fait émerger plusieurs obstacles vécus par les enseignantes dans la transposition de la démarche en classe. Ces difficultés tirent leur origine des tensions ressenties par chacune des enseignantes entre d'anciennes manières de faire et l'appropriation et la mise en place progressive d'une nouvelle démarche d'enseignement de l'écriture. Plusieurs enseignantes ont ainsi été confrontées à des questionnements professionnels qui s'inscrivent dans un processus d'évolution des représentations de l'écriture et de son enseignement (voir l'article de Gagnon, Tremblay et Turgeon, dans ce numéro, qui traite précisément de la question du développement professionnel). C'est aussi ce qui teinte leurs façons respectives de s'approprier et de transposer la démarche, comme le font voir les portraits présentés dans la section précédente. Des réflexions professionnelles sont cependant unanimement partagées, notamment en ce qui concerne la question de l'évaluation des textes produits en cercles d'auteurs. II s'agit là d'un des points qui a le plus " dérangé » les enseignantes, en particulier au début de l'année scolaire. Cette question n'avait pas réellement été abordée en amont, puisqu'un des principes des cercles d'auteurs (principe 5) stipule que l'évaluation de la compétence à écrire doit se faire tout au long du processus et non porter uniquement sur le produit fini... C'est pourtant là que les enseignantes rencontrent d'importants blocages. "Comment vais-je faire pour évaluer mes élèves ? ", "Est-ce que je peux les évaluer individuellement alors qu'ils ont reçu l'aide de leurs pairs pour planifier et réviser? ", "Comment vais-je les préparer à l'examen ministériel de $6^{\mathrm{e}}$ année, s'ils écrivent toujours des textes sur le sujet et le genre de leur choix? », etc.

Les enseignantes réalisent, dans la foulée de ces questionnements, l'importance de distinguer la révision (relire le texte et le retravailler pour l'améliorer) et la correction (corriger les erreurs de grammaire et d'orthographe), une distinction qu'elles-mêmes ne faisaient pas clairement avant de participer au projet. En réponse à l'émergence de ce besoin de perfectionnement, le camp littéraire qui a précédé la deuxième année d'expérimentation (2017-2018), a permis d'aborder la question de la révision en profondeur. Les enseignantes ont pu développer alors une connaissance accrue du processus de révision, en intégrant celle-ci dans leurs pratiques d'enseignement, à travers une mise en place plus 


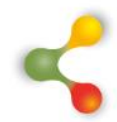

\section{REVUE HYBRIDE DE L'ÉDUCATION}

systématique des cercles de révision en classe. De nombreuses minileçons et plusieurs séances de modelage en révision ont ainsi été proposées par les enseignantes et articulées à la mise en œuvre même de cercles de révision par les élèves. La capacité à réviser des élèves s'en est trouvée améliorée, comme l'illustre l'article de Turgeon, Tremblay et Gagnon dans ce numéro.

Outre la question de la révision, d'autres points propres à la démarche des cercles d'auteurs semblent représenter des défis pour les enseignantes, même après une année d'expérimentation. C'est le cas de la formation des équipes, banale en apparence, mais qui n'en demeure pas moins un enjeu crucial pour une mise en œuvre réussie, puisque tout le travail réalisé dans la démarche repose précisément sur le bon fonctionnement du regroupement d'élèves. C'est au fil d'essais et d'erreurs que les enseignantes trouveront les façons de composer les équipes, mais aussi à travers l'émergence d'une communauté d'auteurs dans la classe que sera facilité le regroupement d'élèves dans les cercles. Les enseignantes remarquent en effet qu'au fil des mois, les élèves développent leurs habiletés coopératives, font preuve de plus en plus de respect et d'écoute entre eux, démontrent une facilité accrue à formuler adéquatement des commentaires pour transmettre leur appréciation ou une suggestion d'amélioration du texte d'un pair, autant d'attitudes et de comportements gagnants pour la formation des équipes de cercles d'auteurs, en conformité avec les principes 7 et 8 de la démarche.

La question du libre choix du sujet représente également un enjeu majeur pour toutes les enseignantes. Une seule d'entre elles aura laissé la liberté du choix du sujet à ses élèves, pour revenir ensuite à des choix imposés. Les enseignantes laissent cependant aux élèves la possibilité de choisir parmi les déclencheurs ou contraintes proposées. Si la créativité doit être au cœur de la démarche (principe 4), ce principe a difficilement été transposé dans les pratiques des enseignantes. La difficulté à lâcherprise, une crainte devant les débordements possibles générés par la liberté de choix, ou la peur que les élèves ne vivent le syndrome de la page blanche sont sans doute en jeu ici... Cela montre pour nous la nécessité d'enseigner comment choisir et développer un bon sujet. C'est d'ailleurs ce qu'on fait les enseignantes lors de la deuxième année du projet, en exploitant plus en profondeur la question de la planification et en faisant vivre de façon plus systématique des cercles de planification à leurs élèves.

Si la mise à l'essai de la première année suscite plusieurs questionnements et pose certains défis, les enseignantes connaissent aussi un grand nombre de réussites. Caroline s'enthousiasme : «Donner le goût d'écrire, c'est fait! ". Les habiletés à formuler des commentaires dans les cercles de partage et dans le cercle de révision se développent progressivement dans toutes les classes. Le recours aux captations vidéos (les élèves se filment eux-mêmes lorsqu'ils vivent un cercle d'auteurs) représente à cet égard une modalité gagnante pour enseigner la démarche 


\section{$\&$}

\section{REVUE HYBRIDE DE L'ÉDUCATION}

et le déroulement attendu des différents types de cercles d'auteurs. Ces vidéos sont utilisées par les enseignantes en classe, en soutien à l'appropriation des phases du processus d'écriture et au bon déroulement de chacune des étapes des cercles d'auteurs.

La mise en œuvre systématique des principes qui fondent la démarche des cercles d'auteurs, point de départ de l'appropriation

Dans notre analyse des caractéristiques de la démarche telle que mise en œuvre durant la première année d'expérimentation, nous constatons chez toutes les enseignantes un engagement fort envers les principes à la base des cercles d'auteurs, en particulier ceux d'écrire fréquemment et de modéliser le processus d'écriture. Le recours à la littérature de jeunesse représente un autre principe clé. En effet, comme le souligne Tauveron (2007), le passage d'une lecture littéraire à une écriture littéraire ne se fait pas directement. L'élève doit pouvoir analyser les œuvres lues avec le soutien et l'étayage de l'enseignant afin de se les approprier, de les comprendre, de les interpréter et d'en dégager ce qui en fait la qualité. De telles façons de lire servent de «tremplin vers l'écriture " (Morin et Montésinos-Gelet, 2007, p. 14) et sont un moyen efficace pour amener les scripteurs à intégrer, dans leurs propres textes, des caractéristiques des textes observés (Harwayne, 1992).

\section{Conclusion}

$\mathrm{Au}$ fil de ce projet, nous avons pu constater la nécessité de disposer de beaucoup de temps, à la fois pour former les enseignantes puis pour les accompagner dans l'appropriation de nouvelles façons d'être et de faire en enseignement de l'écriture. Le fait d'avoir laissé les enseignantes expérimenter la démarche à leur façon, lors de la première année du projet, a représenté une condition de succès, leur permettant d'avancer à leur propre rythme, sans précipiter la mise en œuvre des cercles d'auteurs. La communauté d'apprentissage, dans ses modalités de fonctionnement et à travers la mobilisation des forces respectives de chacun de ses membres a aussi constitué un moteur fort d'avancement et de réussite, comme en témoigne l'article de Gagnon, Tremblay et Turgeon, dans ce numéro.

Nous constatons avec le recul que la démarche des cercles d'auteurs, comme dispositif de formation et de développement professionnel en écriture, est peut-être ce qui a eu le plus eu d'impact sur les pratiques enseignantes lors de la première année du projet. C'est en effet grâce à l'expérience des cercles d'auteurs, vécue pour elles-mêmes à titre d'auteures en herbe (principe 1), que les enseignantes ont progressivement changé leur perception de l'écriture et de son enseignement et ont adapté leurs pratiques en conséquence (Tremblay, Turgeon et Bachand, sous presse). Ce résultat va dans le sens des recommandations formulées par (Locke et Myhill, 2007) à l'effet 


\section{8}

\section{REVUE HYBRIDE DE L'ÉDUCATION}

d'encourager des pratiques d'écriture chez les enseignants pour améliorer l'enseignement de l'écriture.

L'appropriation des principes qui sous-tendent la démarche des cercles d'auteurs, et dont les enseignantes ont fait l'expérience à travers le dispositif d'accompagnement mis en œuvre, représente pour nous l'élément le plus saillant de cette première année d'expérimentation. II est d'abord nécessaire d'adhérer aux principes qui fondent la démarche, et d'arriver à les mettre en œuvre, afin d'instaurer efficacement la démarche dans toutes ses étapes.

La démarche exige enfin une grande part de confiance de la part des enseignants, envers les capacités des élèves. En effet, vivre les cercles d'auteurs veut dire consacrer beaucoup moins de temps à l'enseignement «traditionnel» de l'écriture. Dans la démarche, l'enseignement se fait essentiellement à travers les mini-leçons qui précèdent habituellement les cercles d'auteurs. Les apprentissages des élèves s'appuient sur ces moments d'enseignement explicite et sur la collaboration entre pairs durant chacune des étapes du processus d'écriture. La démarche s'inscrit ainsi dans une vision socioconstructiviste de l'apprentissage à laquelle les enseignantes ont adhéré progressivement, notamment lorsqu'elles ont constaté, d'une part, les capacités effectives des élèves à fonctionner en cercles d'auteurs et, d'autre part, les progrès de ces derniers en écriture à travers l'expérience des cercles d'auteurs et le soutien des autres élèves.

On peut ainsi conclure en disant que les cercles d'auteurs contribuent à la création de véritables communautés d'apprentissage, à l'intérieur desquelles le respect mutuel, la bienveillance et le plaisir d'écrire et de partager ses productions en confiance deviennent des moteurs pour l'apprentissage de l'écriture et le développement corollaire de connaissances et d'habiletés en lecture et en oral. Afin que de telles communautés se développent, les enseignants ont un rôle clé à jouer. Comme l'illustre notre analyse de l'appropriation de la démarche des cercles d'auteurs par les enseignantes, il est nécessaire de disposer de suffisamment de temps pour s'approprier de nouvelles façons d'enseigner. Dans ce processus de développement professionnel singulier, c'est le fait d'avoir vécu la démarche elles-mêmes et d'avoir développé une posture d'auteur (principe 1) qui a guidé en particulier la mise en œuvre des cercles d'auteurs. Chaque enseignante a enfin adhéré de façon plus personnelle à l'un ou l'autre des principes: Caroline a misé sur la fréquence d'écriture comme moyen de soutenir la réalisation des cercles (principe 2) ; Émilie a valorisé l'enseignement de la démarche et des connaissances en écriture (principe 3) ; Brigitte a développé un climat de confiance et de respect (principes 7 et 8), indispensable à la mise en œuvre de la démarche; et Nathalie a valorisé le processus d'écriture dans son ensemble plutôt que de fixer ses intentions pédagogiques et ses pratiques d'évaluation sur le produit fini (principe 5). Nous croyons que cette adhésion et cette mise en 


\section{8}

\section{REVUE HYBRIDE DE L'ÉDUCATION}

pratique des différents principes qui fondent la démarche des cercles d'auteurs représentent des éléments clés de l'appropriation de la démarche par les enseignantes. Des projets de recherche futurs pourront faire appel à ce dispositif d'accompagnement en enseignement de l'écriture, puis, pour compléter les résultats présentés ici, examiner les retombées des changements professionnels observés sur les résultats des élèves en écriture.

\section{Références}

Bautier, E. et Bucheton. D. (1995). L'écriture : qu'est-ce qui s'enseigne, qu'est-ce qui s'apprend, qu'est-ce qui est déjà-là? Le français aujourd'hui, 111, 26-35.

Bhérer, É. (sous presse). Enseigner à réviser grâce aux cercles d'auteurs. Revue Hybride de l'Éducation.

Bing, É. (1976). Et je nageai jusqu'à la page. Édition des femmes.

Blain, S. (2001). Study of Verbal Peer Feedback on the Improvement of the Quality of Writing and the Transfer of Knowledge in Francophone Students in Grade 4 Living in a Minority Situation in Canada. Language, Culture and Curriculum, 14(2), 156-170.

Blain, S. et Painchaud, G. (1999). L'impact de la rétroaction verbale des pairs sur l'amélioration des compositions des élèves de $5 e$ année en immersion française. The Canadian modern language review / La revue canadienne des langues vivantes, 56(1), 73-98.

Blau, S. (1988). Teacher development and the revolution in teaching. English Journal, 2, 30-35.

Bronckart, J.P. (2006). Activités langagières, textes et discours. Pour un interactionnisme sociodiscursif. Delachaux et Niestlé.

Brooks, G.W. (2007). Teachers as readers and writers and as teachers of reading and writing, The Journal of Educational Research, 100(3), 177-191. DOI : 10.3200/JOER.100.3.177-191.

Bucheton, D. (2014). Refonder l'enseignement de l'écriture : Vers des gestes professionnels plus ajustés du primaire au lycée. Editions RETZ.

Buchs, C., Darnon, C., Quiamzade, A., Mugny, G. et Butera, F. (2008). Conflits et apprentissage. Régulation des conflits sociocognitifs et apprentissage. Revue française de pédagogie, 163, 105-205. 


\section{REVUE HYBRIDE DE L'ÉDUCATION}

Cairney, T. et Langbien, S. (1989). Building communities of readers and writers. The Reading Teacher, 42, 560-567.

Calkins, L. (2017). A guide to the writing workshop: Primary grades. Heinemann.

Clanché, P. (1988). L'enfant écrivain : Génétique et symbolique du texte libre. Éditions du Centurion.

Cohen, E.G., Lotan, R.A., Abram, P.L., Scarloss, B.A. et Schultz, S.E. (2002). Can groups learn?. Teachers College Record, 104(6), 1045-1068

Colognesi, S. et Lucchini, S. (2018). Enseigner l'écriture : l'impact des étayages et des interactions entre pairs sur le développement de la compétence scripturale. Revue canadienne de l'éducation, $41(1), 223-249$.

Cotton, K. (1988). Teaching composition : Research on effective practices. Northwest Regional Educational Laboratory : Portland, Oregon.

Cremin, T. (2006). Creativity, uncertainty and discomfort: teachers as writers. Cambridge Journal of Education, 36(3), 415-433.

Daniels, H. (2006). What's the next big thing with literature circles?. Voices from the Middle, 13(4), 10-15.

Dionne, L, Lemyre, F. et Savoie-Zajc, L. (2010). Vers une définition englobante de la communauté d'apprentissage comme dispositif de développement professionnel. Revue des Sciences de l'éducation. 36(1), 25-43.

Doquet-Lacoste, C., Lumbroso, O. et Tauveron, C. (dir.). (2009). Écrire avec, sur, de la littérature. Institut national de recherche pédagogique (INRP).

Dumais, C. (2012). L'enseignement explicite des stratégies d'écoute. Québec français, 164, 57-58.

Fletcher, R. (1993). What a Writer Needs. Heinemann.

Gagnon, B., Tremblay, O. et Turgeon, E. (sous presse) Des enseignantes engagées dans une communauté d'apprentissage sur les cercles d'auteurs : diverses retombées observées sur leur développement professionnel en enseignement de l'écriture. Revue Hybride de l'Éducation. 


\section{REVUE HYBRIDE DE L'ÉDUCATION}

Gilbert, J. et Graham, S. (2010). Teaching writing to elementary students in grades 4-6 : A National survey. The elementary school journal, 110(4), 494-518.

Graham, S., Harris, R. et Mason, L. (2005). Improving the writing performance, knowledge, and self-efficacy of struggling young writers: The effects of self-regulated strategy development. Contemporary educational psychology, 30, 207-241.

Graves, D. (1983). Writing: Teachers and children at work. Heinemann.

Graves, D. (1994). A Fresh Look at Writing. Heinemenan.

Graves, D. (2004). What I've Learned from Teachers of Writing. Language Arts, 82(2), 88-94.

Griffith, R.R. (2010) Students Learn to Read Like Writers: A Framework for Teachers of Writing. Reading Horizons, 50(1), 49-66.

Grossman, P., Valencia, S., Evans, K., Thompson, C., Martin, S. et Place, N. (2000). Transitions into teaching: Learning to teach writing in teacher education and beyond. Dans C.M. Roller (dir.) Learning to teach reading (p. 80-99). International Reading Association.

Gunnery, S. (2007). The writing circle. Pembroke Publishers.

Harste, J.C., Short, K.G. et Burke, C.L. (1988). Creating classrooms for authors. The reading-writing connection. Heinemann Educational Books.

Harward, S., Peterson, N., Korth, B., Wimmer, J., Wilcox, B., Morrison, T.G, Black, S., Simmerman, S. et Pierce, L. (2014). Writing instruction in elementary classrooms: Why teachers engage or do not engage students in writing. Literacy Research and Instruction, 53(3), 205224.

Harwayne, S. (1992). Lasting impressions: Weaving literature into the writing workshop. Heinemann Educational Books.

Hayes, J.R. (1995). Un nouveau modèle du processus d'écriture. Dans J.Y. Boyer, J.-P. Dionne et P. Raymond (dir.), La production de textes : vers un modèle d'enseignement de l'écriture (p. 49-72). Les Éditions Logiques.

Hayes, J.R. et Flower, L.S. (1980). Identifying the organization of writing processes. Dans L.W. Gregg et E.R. Steinberg (dir.), Cognitive processes in writing (p. 3-30). Lawrence Erlbaum Associates. 


\section{REVUE HYBRIDE DE L'ÉDUCATION}

Kolb, D. A. (1984). Experiential learning : Experience as the source of learning and development. Prentice-Hall.

Lafont-Terranova, J. (2009). Se construire à l'école, comme sujet-écrivant : l'apport des ateliers d'écriture. Presses de l'Université Namur

Lamb, V., Plante, I. et Tremblay, O. (2017). Rapport à l'écriture littéraire et pratiques déclarées d'enseignants du primaire en écriture et en lecture littéraires. Nouveaux cahiers de la recherche en éducation, 20(2), 72-98.

Lamblin, A. (2012). Qu'est-ce que la créativité ? Comment la développer? Pour quelles finalités? Cycle 1 [mémoire de maitrise, Université d'Artois]. Institut Universitaire de Formation des Maîtres. https://dumas.ccsd.cnrs.fr/dumas-00735191.

Laroui, R., Morel, M. et Leblanc, S. (2014). Des pratiques pédagogiques de l'enseignement du lire/écrire, déclarées par des enseignantes du primaire. $\quad$ Phronesis, 11-2), 120. https://doi.org/10.7202/1024594ar

Lebrun, M. (2007). Rapport à l'écriture, posture auctoriale et ouverture culturelle. Revue des sciences de l'éducation, 33(2), 383-399. https://doi.org/10.7202/017883ar

Lekeuche, P. (2011). Création, sublimation, idéalisation. Cahiers de psychologie clinique, 1(36), 19-33.

Le Goff, F. (2018). L'écriture de la variation : ses principes. Dans F. Le Goff, et V. Larrivé, Le temps de l'écriture: Écritures de la variation, écritures de la réception (p. 17-37). UGA Éditions.

Le Goff, F. et Larrivé, V. (2018). Le temps de l'écriture : Écritures de la variation, écritures de la réception. UGA Éditions.

Locke, T. et Myhill, D. (2007). Composition in the English/literacy classroom, English Teaching: Practice and Critique, 6(1), 1-10.

May, H. (2004). The impact of America's choice on writing performance in Georgia: First-year results. University of Pennsylvania,

Consortium for Policy Research in Education.

McDonald, J.P., Buchanan, J. et Sterling, R. (2004). The National Writing Project: Scaling up and scaling down. Dans T. K. Glennan, S. J. Bodilly, J. Galegher et K. A. Kerr (dir.), Expanding the reach of 


\section{REVUE HYBRIDE DE L'ÉDUCATION}

education reforms: Perspectives from leaders in the scale-up of educational interventions (p. 81-106). The RAND Corporation.

Morin, M.-Fr. et Montésinos-Gelet, I. (2007). Effet d'un programme d'orthographes approchées en maternelle sur les performances ultérieures en lecture et en écriture d'élèves à risque. Dans Revue des sciences de l'éducation, XXXIII (3), 663-683.

Murray, D.M. (1989). Expecting the unexpected: Teaching myself- and others-to read and write. Boynton/ Cook.

Nadon, Y. (2011). Lire et écrire en première année et pour le reste de sa vie (2e éd.). Chenelière Éducation.

Olness, R. (2005). Using literature to enhance writing instruction . International Reading Association.

Oriol-Boyer, C. (1986). L'art de l'autre: Didactique du texte et communication. Langue française, 70, 45-62.

Oriol-Boyer, C. (1980). Lire pour écrire. Atelier d'écriture et formation des maîtres. Pratiques : linguistique, littérature, didactique, 26, 94-112.

Paillé, P. et Mucchielli, A. (2016). L'analyse qualitative en sciences humaines et sociales ( $4^{\mathrm{e}}$ éd.). Armand Colin.

Ponsot, M. et Deen, R. (1982). Beat not the poor desk: Writing - What to teach, how to teach it and why. Boynton/Cook.

Rainville, B. (sous presse). L'importance d'instaurer un climat de confiance pour profiter pleinement de la démarche des cercles d'auteurs. Revue Hybride de l'Éducation.

Ray, K.W. (2000). Wondrous words: Writers and writing in the elementary classroom. National Council of Teachers of English.

Rebière, M. (2001). Une notion venue d'ailleurs... la posture. Dans Bernié, J.-P. (dir.) Apprentissage, développement et significations (p. 191207). Presses universitaires de Bordeaux.

Reuter, Y. (2002). Enseigner et apprendre à écrire. Construire une didactique de l'écriture. (2e éd.). ESF.

Rief, L. (2003). 100 quickwrites. Scholastic Teaching Resources. 


\section{REVUE HYBRIDE DE L'ÉDUCATION}

Rog, L.J. (2011). 40 nouvelles mini-leçons efficaces pour enseigner l'écriture. Chenelière Éducation.

Routman, R. (2010). Enseigner l'écriture : revenir à l'essentiel. Chenelière Éducation.

Schultz, K. (1997). "Do You Want to Be in My Story?" : Collaborative writing in an urban elementary classroom. Journal of Literacy Research, 29(2), 253-287.

Shea, M. (2015). Differentiating writing instruction: meeting the diverse needs of authors in a classroom. Journal of inquiry and action in education, 6(2), 80-118.

Short, K.G., Harste, J.C. et Burke, C.L. (1996). Creating classrooms for authors and inquirers. Heinemann Educational Books.

Simard, C. (1999). L'annotation des textes d'élèves. Québec français, 115, 32-38.

Slavin,R. E. (1980). Cooperative learning. Review of Educational Research, 50, 315-342.

Sorin, N. (2005). Vers une didactique de l'écriture littéraire du récit de fiction au primaire. Nouveaux cahiers de la recherche de l'éducation, 8(1), 65-78.

Steineke, N. (2002). Reading and writing together: Collaborative literacy in action. Heinemann Educational Books.

Tauveron, C. (2002). L'écriture littéraire : une relation dialectique entre intention artistique et attention esthétique. Repères, 26(1), 203215.

Tauveron, C. (2007). Le texte singulier de l'élève ou la question du sujet scripteur. Le français aujourd'hui, 157(2), 75-82.

Tauveron, C. et Sève, P. (2005). Vers une écriture littéraire : ou comment construire une posture d'auteur à l'école de la GS au CM. Hatier.

Tremblay, O. et Turgeon, E. (2019). Collaboration dans les cercles d'auteurs : pistes pour travailler la planification. Vivre le primaire. 32(1), 11-13. 


\section{REVUE HYBRIDE DE L'ÉDUCATION}

Tremblay, O., Turgeon, E. et Bachand, V. (sous presse). Six enseignantes en quête d'une posture d'auteure. Dans A. Pauzet (dir.), Les écritures créatives en formation et dans le monde professionnel. Représentations contemporaines, nouveaux enjeux, transferts de compétences. Presses Universitaires de Rennes.

Turgeon, E., Tremblay, O. et Gagnon, B. (sous presse). Améliorer son texte grâce aux cercles d'auteurs : regard sur la compétence à réviser d'élèves de 6e année. Revue Hybride de l'Éducation.

Vopat, J. (2009). Writing circles: Kids revolutionize workshops. Heinemann Educational Books.

Whyte, A., Lazarte, A., Thompson, I., Ellis, N., Muse, A. et Talbot, R. (2007). The national writing project, teachers' writing lives, and student achievement in writing. Action in Teacher Education, 29(2), 5-16. 